\title{
Organ chips, organoids and the animal testing conundrum
}

\author{
Nature Reviews Materials speaks to Donald Ingber, Founding Director of the Wyss Institute for Biologically Inspired \\ Engineering at Harvard University, about the animal testing conundrum and the importance of human-relevant \\ models in biomedical research.
}

\begin{abstract}
Q Animal models are commonly used to study human diseases and treatments; however, they are often limited in their ability to mimic human conditions, in particular, on the molecular and cellular levels. Why are we still using them?

The first and short answer is that we use them because they are there, and because we have used them in the past and we know how to work with animal models. Secondly, many in vitro models are too simplified to predict in vivo conditions. A main power of animal models is the possibility of genetic engineering, based on specific genetic alterations that we suspect may be involved. The question is whether we are fooling ourselves, because we convince ourselves that what we see is what we thought it should be. Indeed, many drugs first go through animal models before reaching clinical trials, and the large majority of those drugs fail in the clinic. Of course, there are also ethical considerations when it comes to animal models.

But we are now at a point at which we have much more complex in vitro models available, ranging from organoids to multiphysiological systems and organs-on-chips. Some of these in vitro models are as good or even better than animal models. However, these platforms need to be fit-for-purpose, because they are not generic. There is no in vitro model that can replace all animal models. You need different models even for one organ, such as the lung. One would require distinct models for asthma, pulmonary fibrosis or pulmonary edema, for example. We work with animal models, because they often exhibit phenotypes that appear like those of a human disease, but the underlying molecular and cellular mechanisms can be quite different. Moreover, some technologies, such as therapeutic CRISPR RNAs or monoclonal
\end{abstract}

antibodies do not work in animal models at all, because they are so specific for human target sequences. Similarly, vaccine responses are often not representative in animal models, not even in non-human primates.

However, for a long time there was really no alternative to animal models. Of course, we need to study animal models because of one-health applications, linking animal health, human health and the environment. However, you can also create animal organs-on-chips, such as a bat intestine-on-a-chip. In this way, zoonotic infections, such as COVID-19, can also be studied using in vitro platforms, and you can also undertake genetic engineering in organs-on-chips. We are now at a time, in which we can create in vitro systems that are more relevant to humans or animal models, depending on how we build them.

\section{Q Many publishers, funding agencies and} regulatory agencies often dictate the use of animal models at high numbers. Do you think that these requirements should be challenged? Absolutely. I think if reviewers were aware that there are alternative models out there, they might demand validation in a human-relevant model rather than in an animal model. Many reviewers, however, do not know about these new in vitro models, such as human organs-on-chips or other types of complex microphysiological systems. Reviewers often make valid points, for example, to consider the organism-level context or pharmacokinetics; however, an animal model may often not be the best model to study these aspects. For example, we found that breathing motions influence the rate of lung cancer growth and invasion in a human lung chip. In this case, it would have been impossible to use an animal model, because, obviously, we cannot keep an animal alive for three weeks without

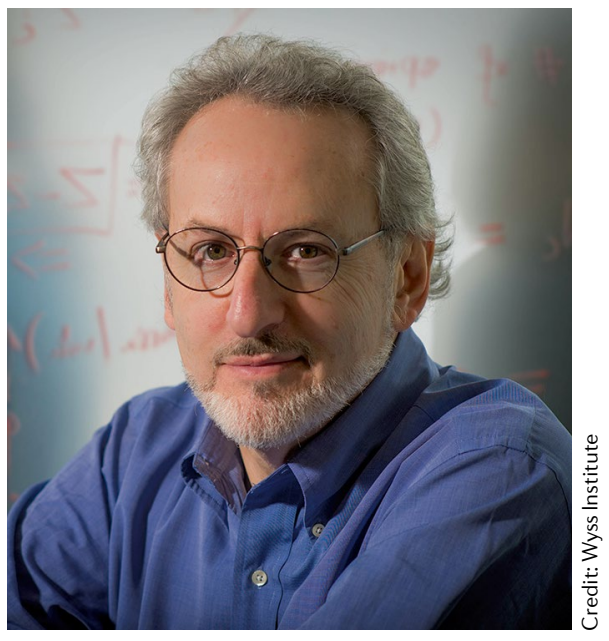

breathing. However, this was demanded by one reviewer.

Asking for animal models is often a reflex, because this is how it's been done for many years, even in cases, in which animal models are known to not replicate human physiology or pathophysiology. It is important to ask what the most important human-relevant experiment is, and if there is an in vitro model available that has been validated for human clinical mimicry, it should be valid to use this model. In my past discussions, even the FDA suggested that in vitro models could be useful if they are equally good as animal models, and not necessarily better, provided they are human and equally robust and validated. The same criteria should also be applied by reviewers for grants and papers.

\section{Q. Do you think there is barrier to using in vitro platforms instead of animals, in particular, for researchers who are not engineers? \\ People tend to do what they were trained to do, and they use methods they are comfortable with. It takes energy to learn}


cas ... we are now at a point at which we have much more complex in vitro models available, ranging from organoids to multiphysiological systems and organs-on-chips. Some of these in vitro models are as good or even better than animal models

about new technologies, their nuances and limitations. However, nowadays, you really do not need to know engineering to use organ-on-a-chip systems. There are even commercial suppliers. But you do need to adapt to these new systems, maybe run further controls to compare with previous data and to ensure that they are robust. So, yes, there is an activation barrier to using new in vitro platforms. Basically, we need to change the way we do things.

\section{Q You have developed and worked on organ-on-a-chip systems for many years, and there are now chips available with very high complexity and human mimicry. However, the lack of immune components and systemic investigation is often criticized. Do you think this is valid criticism? And how could it be addressed?}

I want to give an example about incorporation of immune responses. We worked on pulmonary edema induced by interleukin-2; it's dose-limiting toxicity is pulmonary vascular leakage, which we mimicked on a chip. We clearly could show interleukin-2-induced edema without the need of immune cells, which is something you cannot do in an animal model or in a human clinical trial.

Similarly, you can use chips to decouple effects of mechanics and fluid flow. This is essentially a synthetic biology approach at the cell, tissue and organ level that allows us to apply a sort of Einsteinian methodology; that is, identifying the simplest systems that can recapitulate the in vivo complexity of the question you are interested in. For example, if I am interested in epithelial-endothelial crosstalk, I do not necessarily need immune cells.
But of course, we can add immune cells and other components to organs-on-a-chip platforms and we do when we cannot replicate a phenotype without them. For example, you can add circulating immune cells and show recruitment and shear-stress dependent initial adhesion to the endothelium lining our chips. Indeed, I see this as one of the big advantages of these systems. We can add components in one-at-a-time and control them independently. For example, we made a lymphoid follicle-on-a-chip and tested an influenza vaccine. We could replicate germinal centre formation, antibody class switching and IgG production and we detected the same cytokines as seen in humans who received the same commercial vaccine. We are now also testing the effects of new immune adjuvants in these platforms.

Similarly, we have developed a bone marrow-on-chip, which contains the hematopoietic lineage. Other groups have also incorporated immune cells in organ-on-a-chip systems. Importantly, if you can replicate physiology with a simple system, you've got a handle on control.

The power of the microfluidic organs-onchips we study is that they provide a window on molecular scale activities inside living cells in a physiologically relevant organ context, including tissue-tissue interfaces and relevant cells, as well as fluid flow, air-liquid interfaces and mechanical motions.

Also, using organs-on-chips you can visualize and analyse the same location again and again over time. This is not possible in more complex models, such as organoids or animal models. I see organs-on-chips as living 3D cross-sections of major functional units of human or animal organs, which we can analyse over time. We can even culture living microbiome in contact with living human cells using organs-on-chips. The discovery of the importance of the microbiome is one of the major paradigm shifts in medicine over the last 15 years, and you cannot study the human microbiome in animals, because they have a different microbiome.

So in terms of your initial question, it all comes down to whether your mechanism involves an immunological response.
Then, of course, you need to add the relevant components. If you are not sure, it should be one of the variables you explore. But if you know what your response is, and you start simple and mimic the response with high fidelity early on, then you may not need greater complexity.

Q What are the next important milestones in organs-on-chips, in particular, from a materials science and engineering perspective?

Some of the biggest challenges are the need for more online read-outs and sensors. The idea is to instrument chips so that you can get multiple read-outs of functionality, in particular, cellular, biochemical and even genetic readouts. Multiplexed readouts in real time would change the field.

In addition, higher throughput is needed, with the possibility to analyse multiple individual channels in parallel; also, visualization at high resolution and automated molecular analysis in small volumes. We need to get the most information out of these systems in real time.

Regarding materials science, there is certainly a need for better materials that are optically clear, flexible and biocompatible. We use polydimethylsiloxane (PDMS), because it is simple, cheap and gas-permeable, but it also can absorb certain small drugs. So, alternatives would be welcome. Moreover, coming up with new ways to cheaply mass fabricate these chips is important for commercialization. Similarly, techniques to bond material layers is critical for instrumenting chips to prevent materials from peeling apart in fluid flow.

There is also a proposal to start a new institute in the National Institutes of Health (NIH) focusing on in vitro models, which may help to build up the number of people familiar with this opportunity. This should help to achieve a critical mass of researchers using this technology, who will then act as reviewers of grants and papers. Engaging more researchers may help to push the technology over the top, such that people start looking beyond animal models.

Interviewed by Christine Horejs

https://doi.org/10.1038/s41578-021-00313-z 